\title{
MELK inhibition targets cancer stem cells through downregulation of SOX2 expression in head and neck cancer cells
}

\author{
LILI REN $^{1,2}$, BOYA DENG ${ }^{1}$, VASSILIKI SALOURA ${ }^{1}$, JAE-HYUN PARK $^{1}$ and YUSUKE NAKAMURA ${ }^{1,3}$ \\ ${ }^{1}$ Department of Medicine, The University of Chicago, Chicago, IL 60637, USA; \\ ${ }^{2}$ Cytotherapy Laboratory, Shenzhen People's Hospital, The Second Clinical Medical College of Jinan University, \\ Shenzhen, Guangdong 518020, P.R. China; ${ }^{3}$ Department of Surgery, The University of Chicago, \\ Chicago, IL 60637, USA
}

Received May 10, 2018; Accepted December 17, 2018

DOI: $10.3892 / o r .2019 .6988$

\begin{abstract}
Maternal embryonic leucine zipper kinase (MELK) has been reported to serve critical roles in the maintenance of stemness of cancer cells, although its mechanism remains unclear. Since SRY-box 2 (SOX2) was demonstrated to be involved in self-renewal and tumorigenicity of head and neck squamous cell carcinoma (HNSCC) and is aberrantly expressed in HNSCC tumors, the association between MELK and SOX2 was examined. Firstly, MELK inhibition was performed by small interfering RNA or MELK inhibitor OTS167, and it was determined that MELK inhibition by these approaches could decrease the SOX2 expression in HNSCC cells and OTS167 could suppress the SOX2 expression in a dose-dependent manner. The present results indicated that MELK inhibition may target cancer stem cells (CSCs) through downregulation of the SOX 2 gene. To further confirm the transcriptional regulation of SOX2, the transcription factors (TFs) were screened for SOX2 using a promoter-binding TF assay followed by reverse transcription-quantitative polymerase chain reaction and a decrease of the majority of the SOX2 TFs following MELK knockdown was observed. The present results provide evidence that MELK serves a key role in CSCs through the regulation of SOX2 and further indicates that MELK inhibition may also be promising for clinical applications in the treatment of HNSCC.
\end{abstract}

Correspondence to: Professor Yusuke Nakamura, Department of Medicine, The University of Chicago, 900 E 57th Street, Chicago, IL 60637, USA

E-mail: yusuke.nakamura@jfcr.or.jp

Abbreviations: MELK, maternal embryonic leucine zipper kinase; HNSCC, head and neck squamous cell carcinoma; CSC, cancer stem cell

Key words: cancer stem cell, kinase inhibitors, head and neck cancer, MELK, SRY-box 2

\section{Introduction}

Head and neck squamous cell carcinoma (HNSCC) is the sixth most common cancer type with poor prognosis globally from 2005-2015 (1). Despite advances in its diagnosis and treatment, the 5-year survival rate (1983-2002) of patients with late stage HNSCC globally was $30-50 \%$ and has improved slightly in recent decades (2). Therefore, development of novel therapies with high efficacy and low toxicity is required.

Maternal embryonic leucine zipper kinase (MELK) has been reported to serve critical roles in cancer cell proliferation and maintenance of stemness (3). It was previously reported that MELK is a promising target for cancer therapy and we developed a potent MELK inhibitor, OTS167, which exhibited effective growth suppression in mice xenograft models of a number of cancer types, including breast, lung, prostate, and pancreas cancer $(4,5)$. At present, the therapeutic potential of OTS167 is being evaluated in clinical trials, including NCT01910545 (6), NCT02795520 (7) and NCT02926690 (8).

Cancer stem cells (CSCs) are more resistant to chemotherapy/radiation than non-stem cancer cells and are considered to serve important roles in cancer recurrence and/or metastasis (9). Numerous studies for targeting CSCs have purported pluripotency-associated transcription factor (TF) SRY-box 2 (SOX2) to be a therapeutic target due to its gene amplification and/or overexpression in cancer cells (10-12). The SOX family is a group of TFs that have been demonstrated to have critical roles in developmental and stem cell biology (13). The SOX2 gene is located on chromosome 3q26.3-q27, belongs to the SOXB1 group and encodes for a protein consisting of 317 amino acids (14). The aberrant expression of SOX2, which promotes cellular proliferation, invasion, migration, metastasis and evading apoptotic signals, has been reported in a number of human cancer types, including lung, esophageal, pancreatic, breast, ovarian and hepatocellular carcinoma, as well as head and neck cancer (HNSCC) (15-19).

In HNSCC, SOX2 was demonstrated to regulate selfrenewal and tumorigenicity of HNSCC stem-like cells (20). Ectopic expression of SOX2 induced cell proliferation and enhanced stemness-associated features (20). Knockdown of 
SOX2 in HNSCC CSCs attenuated their self-renewal capacity, chemoresistance, invasiveness and in vivo tumorigenicity (18). Meta-analysis to examine associations between SOX2 expression levels and clinicopathological/prognostic parameters in HNSCC of 7 studies ( 9 cohorts) indicated that the high SOX2 expression was strongly associated with unfavorable 5 -year overall survival [hazard ratio (HR), 1.54; $95 \%$ confidence interval (CI), 1.09-2.18] and disease-free survival rate (HR, $1.54 ; 95 \% \mathrm{CI}, 1.13-2.10)$ of patients (21-27). Additionally, increased SOX2 expression was also significantly associated with a high tumor grade and an advanced TNM stage as well as metastatic lymph-node status and distant metastasis (28).

In the present study, the effect of MELK knockdown and inhibition of MELK enzymatic activity by its inhibitor, OTS167, on SOX2 expression in HNSCC cells was reported. The data indicated that the MELK inhibitor may be a promising modality for the treatment of HNSCC.

\section{Materials and methods}

Cell lines. The HNSCC cell lines UD-SCC-2, UT-SCC-40, HN-SCC-151, FaDu, JSQ-3, HN-5, HN-6 and HN13 were provided by Dr Tanguy Y. Seiwert (Department of Medicine, University of Chicago, Chicago, IL, USA). The YD-10B cell line was purchased from the Korean Cell Line Bank (KCLB; KCLB no. 60503; Korean Cell Line Research Foundation, Seoul, Korea). UD-SCC-2, HN-SCC-151, JSQ-3 and UT-SCC-40 cells were cultured in Dulbecco's modified Eagle's medium (DMEM)/F12 (Life Technologies; Thermo Fisher Scientific, Inc., Waltham, MA, USA) with 10\% FBS (Gemini Bio-Products, West Sacramento, CA, USA) and $1 \mathrm{X}$ antibiotic-antimycotic solution (Sigma-Aldrich; Merck KGaA, Darmstadt, Germany). HN13, HN-5, HN-6 and YD-10B cells were cultured in DMEM (Life Technologies; Thermo Fisher Scientific, Inc.) with 10\% FBS and 1X antibiotic-antimycotic solution. FaDu was cultured in RPMI-1640 media (Life Technologies; Thermo Fisher Scientific, Inc.) with $10 \%$ FBS and $1 \mathrm{X}$ antibiotic-antimycotic solution. All cells were maintained at $37^{\circ} \mathrm{C}$ in humidified air in an atmosphere containing $5 \% \mathrm{CO}_{2}$.

Sphere formation. For the sphere formation assay, $1 \times 10^{3}$ cells of 6 individual HNSCC cell lines (HN13, FaDu, UD-SCC-2, HN-6, YD-10B and HN-5) with a high or undetectable expression level of MELK were seeded onto Ultra-Low attachment $60 \mathrm{~mm}$ plates (Corning Incorporated, Corning, NY, USA) and cultured in corresponding serum-free medium for two weeks at $37^{\circ} \mathrm{C}$ in humidified air in an atmosphere containing $5 \% \mathrm{CO}_{2}$. A total of $500 \mu 1$ fresh medium corresponding to each cell line was added every 3-4 days. The spheres were observed and recorded with an Invitrogen EVOS FL Auto 2 Cell Imaging System under an Evos FL Auto 2 light microscope (x40; Invitrogen; Thermo Fisher Scientific, Inc.).

Western blot analysis and antibodies. A total of 9 cell lines (FaDu, HN13, UD-SCC-2, HN-6, UT-SCC-40, HN-SCC-151, JSQ-3, YD10-B and HN-5) were lysed on ice with IP lysis buffer (Thermo Fisher Scientific, Inc.) containing protease inhibitor cocktail set III $(1: 1,000)$ and phosphatase inhibitor cocktail set I (1:100) (EMD Millipore, Billerica, MA, USA) to evaluate the MELK expression level. HN13 and UT-SCC-40 were used to evaluate the change of SOX2 expression level after MELK inhibition following the same lysis protocol aforementioned. Protein concentration was measured using a BCA assay. A total of $20 \mu \mathrm{g}$ lysate protein were loaded and separated by electrophoresis using an Any kD precast polyacrylamide gel (Bio-Rad Laboratories, Inc., Hercules, CA, USA) and transferred onto a polyvinylidene fluoride (PVDF) membrane. Following blocking with 5\% skim milk (Thermo Fisher Scientific, Inc.) in TBS with 0.1\% Tween-20 (TBST) buffer for $1 \mathrm{~h}$ at room temperature, membranes were incubated with the primary antibodies, anti-MELK monoclonal antibody [1:5,000; produced in-house, as previously described in (29)] or anti-SOX2 monoclonal antibody (1:1,000; cat. no. MA1-014; Thermo Fisher Scientific, Inc.) overnight at $4^{\circ} \mathrm{C}$. Anti- $\beta$-actin antibody (1:10,000; cat. no. A5441; Sigma-Aldrich; Merck KGaA) was used as a loading control and incubated with membranes overnight at $4^{\circ} \mathrm{C}$. After washing three times with TBST buffer, membranes were incubated with rabbit anti-mouse IgG-horseradish peroxidase (HRP) antibody as the secondary antibody (1:1,000; cat. no. Sc358917; Santa Cruz Biotechnology, Inc., Dallas, TX, USA) for $1 \mathrm{~h}$ at room temperature. The expression level of MELK in each cancer cell line was quantified with the ImageJ $1.51 \mathrm{~h}$ software (National Institutes of Health, Bethesda, MD, USA) and normalized with that of $\beta$-actin with GraphPad Prism version 7.01 (GraphPad Software, Inc., La Jolla, CA, USA).

Growth suppressive effect of OTS167 in HNSCC cells. MTT was conducted using Cell Counting Kit-8 (Dojindo Molecular Technologies, Inc., Kumamoto, Japan), according to the manufacturer's protocols, to calculate the half-maximum inhibitory concentration $\left(\mathrm{IC}_{50}\right)$ value of OTS167 on cell lines. In brief, $4 \times 10^{3}$ cells were seeded into 96-well flat plates. Cells treated with graded concentrations of OTS167 were cultured for $72 \mathrm{~h}$ at $37^{\circ} \mathrm{C}$ in an atmosphere containing $5 \% \mathrm{CO}_{2}$. OTS167 was provided by OncoTherapy Science, Inc. (Kawasaki, Japan). UD-SCC2-2, HN13 and FaDu cells, which represent cell lines with high MELK expression level, were treated with OTS167 at concentrations of $0,0.39,0.78,1.56,3.12,6.25,12.5,25$, $50,100,500$ or 1,000 nM. UT-SCC-40 cells and JSQ-3 cells represent cell lines with moderate MELK expression level. UT-SCC-40 cells were treated with OTS167 at concentrations of $0,1.2,2.4,4.9,9.8,19.5,39,78,156,312,625$ or $1,000 \mathrm{nM}$. JSQ-3 cells were treated with OTS167 at concentrations of 0 , $0.6,1.2,2.4,4.9,9.8,19.5,39,78,156,312$ or $625 \mathrm{nM}$. YD-10B and HN-5 cells, which represent cell lines with undetectable MELK expression level, were treated with OTS167 at concentrations of $0,0.78,1.56,3.12,6.25,12.5,25,50,100,200$ or $1,000 \mathrm{nM}$. To quantify cell viability, the 96 -well plate was measured at a wavelength of $450 \mathrm{~nm}$ in an iMark microplate absorbance reader (Bio-Rad Laboratories, Inc.) following reaction for $1-4 \mathrm{~h}$ at $37^{\circ} \mathrm{C}$ until the maximum absorbance reached around 1. All these experiments were conducted in triplicate. The $\mathrm{IC}_{50}$ values of OTS167 were calculated with GraphPad Prism version 7.01.

Oligo small interfering RNA (siRNA) and transfection. To knockdown MELK gene expression, HN13 and UT-SCC-40 cells were transfected with 200 pmol oligonucleotide siRNA 
Table I. Primers used in real time RT-PCR and promoter-binding TFs assay.

\begin{tabular}{lll}
\hline Primer & \multicolumn{1}{c}{ Forward } & \multicolumn{1}{c}{ Reverse } \\
\hline$M E L K$ & 5'-GCTGCAAGGTATAATTGATGGA-3' & 5'-CAGTAACATAATGACAGATGGGC-3' \\
SOX2 & 5'-TCGGCGCCGGGGAGATACAT-3' \\
$C / E B P$ A & 5'-CCCCCGGCGGCAATAGCA-3' & 5'-TCATAACTCCGGTCCCTCTG-3' \\
$C / E B P B$ & 5'-TGTATACCCCTGGTGGGAGA-3' & 5'-AGCTGCTCCACCTTCTTCTG-3' \\
$P b x 1$ & 5'-GACAAGCACAGCGACGAGTA-3' & 5'-CTCTTTGGCTTCCTTCACTGG-3' \\
$N k x 2-5$ & 5'-CAGATGCAGCTCAAGCAGAG-3' & 5'-CTCATTGCACGCTGCATAAT-3' \\
SMUC & 5'-CTCAACAGCTCCCTGACTC-3' & 5'-GTCGTTCAGGACACAGCAGA-3' \\
SOX9 & 5'-GGCCACACACTGTCTCCAC-3' & 5'-CTCCTCAAGGTCGAGTGAGC-3' \\
SOX2-promoter & 5'-TACGACTACACCGACCACCA-3' & 5'-GGGTTTCTAGCGACCAATCA-3' \\
GAPDH & 5'-TGAGAGAGTGTTGGCACCTG-3' & 5'-GGTTGAGCACAGGGTACTTTATT-3' \\
\hline
\end{tabular}

MELK, maternal embryonic leucine zipper kinase; SOX2, SOX2, SRY-box 2; C/EBP, CCAAT enhancer binding protein; SMUC, snail family transcription repressor 3 .

using Lipofectamine ${ }^{\circledR}$ RNAiMAX (Invitrogen; Thermo Fisher Scientific, Inc.), following the manufacturer's protocols. The target sequence of oligo siMELK (200 pmol; Sigma-Aldrich, Merck KGaA) was 5'-GACAUCCUAUCUAGCUGCA-3' for MELK. Western blot analysis was conducted to evaluate the efficiency of MELK knockdown with siRNA at the $70 \%$ cell confluency condition and optimize the transfection efficiency, as aforementioned. SIC001 Mission siRNA Universal from Sigma-Aldrich (200 pmol; Merck KGaA) was used as a control (siControl). After $48 \mathrm{~h}$ of transfection, the subsequent analysis was performed.

MELK inhibition by OTS167 treatment. To inhibit MELK in HNSCCs, HN13 and UT-SCC-40 cells were treated with OTS167 dissolved in dimethyl sulfoxide (DMSO) at different concentrations (equivalent to $1 \mathrm{X}, 2 \mathrm{X}, 4 \mathrm{X}$ and $8 \mathrm{X} \mathrm{IC} \mathrm{IC}_{50}$ concentrations) for $48 \mathrm{~h}$ at $37^{\circ} \mathrm{C}$, cells treated by equal amounts of DMSO were used as control.

In vitro kinase assay. MELK recombinant protein was obtained from OncoTherapy Science, Inc. Histone H3 recombinant protein (EMD Millipore, Burlington, MA) was used as a positive control in the in vitro kinase assay. ATP $(10 \mathrm{mM}$; cat. no. 9804) and kinase buffer (10X; cat. no. 9802) were purchased from Cell Signaling Technology, Inc. (Danvers, MA, USA). In each reaction, $0.15 \mu \mathrm{M}$ SOX2 recombinant protein $(0.26 \mu \mathrm{g}$; cat. no. LS-G62-25; LifeSpan Biosciences, Inc., Seattle, WA, USA) or $0.15 \mu \mathrm{M}$ Histone $\mathrm{H} 3$ recombinant protein (0.13 $\mu \mathrm{g}$; cat. no. 14-494; EMD Millipore) were mixed with $0.15 \mu \mathrm{M}$ MELK recombinant protein $(0.29 \mu \mathrm{g})$ in $50 \mu \mathrm{l}$ kinase buffer and incubated for $2 \mathrm{~h}$ at $30^{\circ} \mathrm{C}$. The reaction was terminated by the addition of $1 \%$ SDS sample buffer (Thermo Fisher Scientific, Inc.) and boiled at $70^{\circ} \mathrm{C}$ for $5 \mathrm{~min}$. Finally, the total reacted $50 \mu 1$ samples were electrophoresed on an Any kD precast polyacrylamide gel, transferred onto a PVDF membrane and followed with the standard western blot analysis protocols, as aforementioned. The primary antibody (anti-phospho-Ser/Thr/Tyr antibody; 1:1,000; cat. no. ab15556) was purchased from Abcam (Cambridge, MA, USA) and incubated with membranes overnight at $37^{\circ} \mathrm{C}$ Rabbit anti-mouse
IgG-HRP antibody was used as the secondary antibody (1:1,000; cat. no. Sc358917; Santa Cruz Biotechnology) and incubated for $1 \mathrm{~h}$ at room temperature.

Promoter binding TF profiling plate array. A promoter binding TF profiling plate array (Promoter-Binding TF Profiling Array II; Signosis, Inc., Santa Clara, CA, USA) was performed according to the manufacturer's protocols. Briefly, 96 biotin-labeled TF probes were mixed with nuclear extract of HN13 cells with or without the SOX2 promoter DNA. SOX2 promoter DNA was amplified by Q $5{ }^{\circledR}$ High-Fidelity DNA Polymerase (New England Biolab, Inc., Ipswich, MA, USA) with SOX2 promoter primers (Sigma-Aldrich, Merck KGaA) detailed in Table I and PCR was performed under the following thermocycling conditions: $95^{\circ} \mathrm{C}$ for $10 \mathrm{~min}$, followed by 35 cycles of $95^{\circ} \mathrm{C}$ for $15 \mathrm{sec}, 60^{\circ} \mathrm{C}$ for $30 \mathrm{sec}$ and $72^{\circ} \mathrm{C}$ for $30 \mathrm{sec}$. PCR products was purified with a MinElute Gel Extraction kit (Qiagen, Inc., Valencia, CA, USA). The TF-DNA complex was separated from free probes using an isolation column, and the TF-bound probes were eluted using elution buffer. Hybridization of eluted probes was performed with hybridization plate. The bound probe was detected using a streptavidin-horseradish peroxidase conjugate and measured with a SynergyH4 plate reader (BioTek Instruments, Inc., Winooski, VT, USA). The decreased fold of signal was calculated.

Reverse transcription-quantitative PCR (RT-qPCR). Total RNA was extracted from HN13 and UT-SCC-40 cells using a RNeasy Mini kit (Qiagen, Inc.), and then $2 \mu \mathrm{g}$ RNA was reverse transcribed using a SuperScript III First-Strand Synthesis System (Thermo Fisher Scientific, Inc.), according to the manufacturer's protocols. The reaction system was incubated for $60 \mathrm{~min}$ at $42^{\circ} \mathrm{C}$. RT-qPCR was performed using primers (Sigma-Aldrich, Merck KGaA) and $\mathrm{SYBR}^{\circledR}$-Green Real-Time PCR Master mix (Thermo Fisher Scientific, Inc.) in a ViiA 7 system (Life Technologies; Thermo Fisher Scientific, Inc.). The PCR conditions were as follows: $95^{\circ} \mathrm{C}$ for $10 \mathrm{~min}$ followed by 40 cycles of $95^{\circ} \mathrm{C}$ for $15 \mathrm{sec}$ and $60^{\circ} \mathrm{C}$ for $1 \mathrm{~min}$. The PCR primer sequences were listed in Table I. The 
A
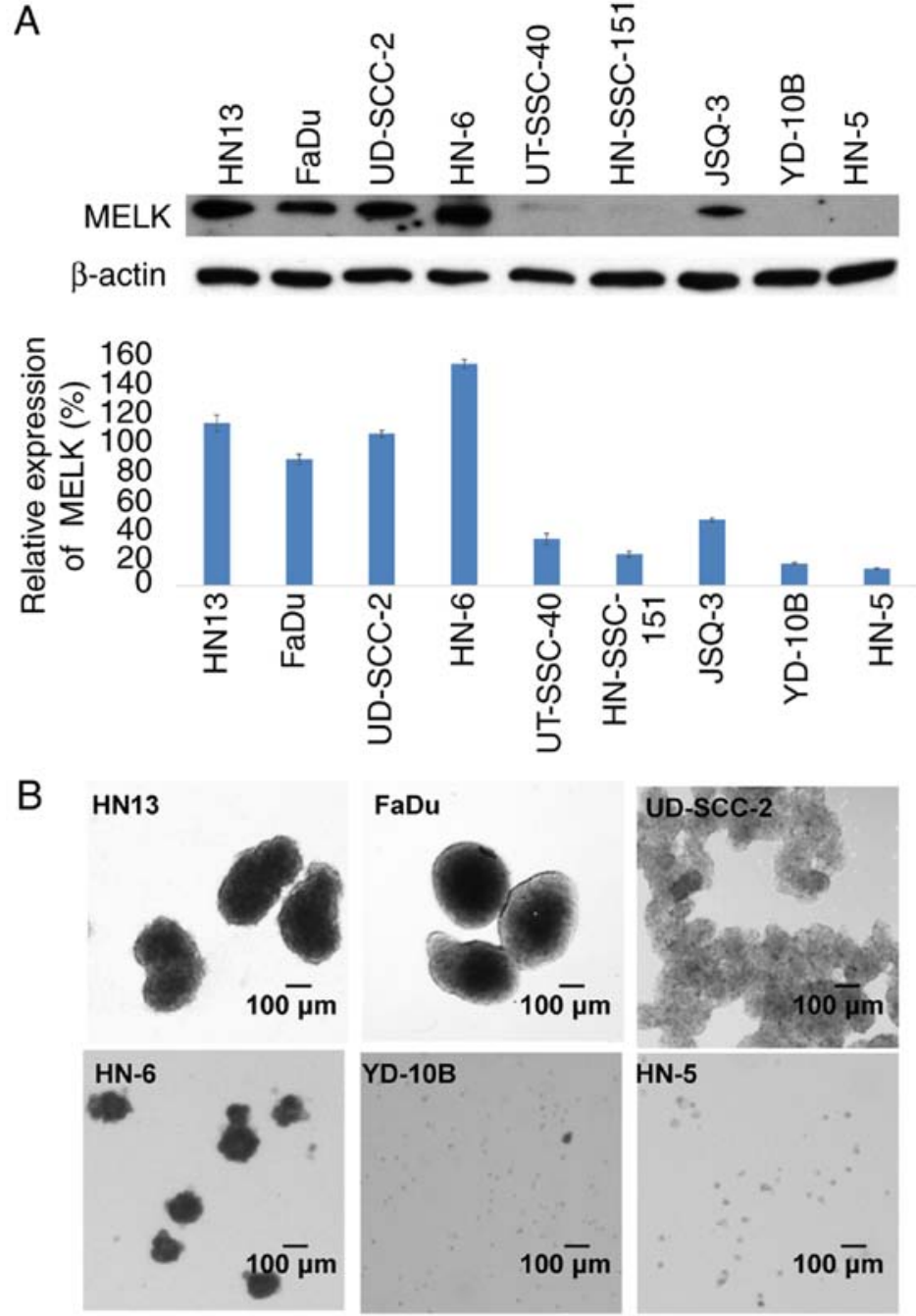

Figure 1. MELK expression levels and sphere formation of HNSCC cell lines. (A) MELK expression of individual HNSCC cell lines. (B) Sphere formation of HNSCC cell lines with different MELK expression levels (scale bar, $100 \mu \mathrm{m}$ ). MELK, maternal embryonic leucine zipper kinase; HNSCC, head and neck squamous cell carcnoma.

expression levels were normalized with that of GAPDH with the $\Delta \Delta \mathrm{Cq}$ method (30).

Statistical analysis. Data analysis was conducted using GraphPad Prism version 7.01. Data are presented as mean \pm standard deviation. Differences between two groups were calculated for significance using Student's t-test and differences between multiple groups were calculated for significance using the analysis of variance analysis followed by Dunnett's multiple comparisons test. $\mathrm{P}<0.05$ was considered to indicate a statistically significant difference.

\section{Results}

Increased MELK expression associates with stronger sphere formation ability. To investigate a role of MELK expression in the stemness of HNSCC cells, the MELK expression levels was examined in YD-10B, FaDu, HN-6, HN13, UT-SCC-40, HN-5, HN-SCC-151, JSQ-3 and UD-SCC-2 cells by normalization with that of $\beta$-actin. The results demonstrated that the relative expression levels of MELK in four HNSCC cell lines, HN13, FaDu, UD-SCC-2 and HN-6, were notably high (113,
87,105 and $154 \%$ of the $\beta$-actin expression level, respectively); whereas, in UT-SCC-40, HN-SCC-151, JSQ-3, YD-10B and HN-5 cells, MELK expression levels were notably reduced $(32,21,46,15$ and $11 \%$ of the $\beta$-actin expression level, respectively), as depicted in Fig. 1A. Subsequent sphere formation experiments of the two-week culture in serum-free medium indicated that the four cell lines, HN13, FaDu, UD-SCC-2 and $\mathrm{HN}-6$, in which MELK had increased expression, formed large spheres, compared with HN-5 and YD-10B cells with reduced MELK expression, which formed small spheres. Additionally, UD-SCC-2 cells formed numerous small spheres that stacked together (Fig. 1B).

Growth suppressive effect of OTS167 in HNSCC cancer cells. As previous reported, a potent MELK inhibitor, OTS167, was developed, which exhibited a significant growth suppressive effect on lung cancer cells (31), kidney cancer cells (32) as well as multiple myeloma cells (33). In the present study, the $\mathrm{IC}_{50}$ values of OTS167 were examined to measure its growth inhibitory effect on seven HNSCC cell lines. This included five HNSCC cell lines with relatively increased MELK expression levels, UD-SCC-2, FaDu, HN13, UT-SCC-40 and JSQ-3 cells, 
A

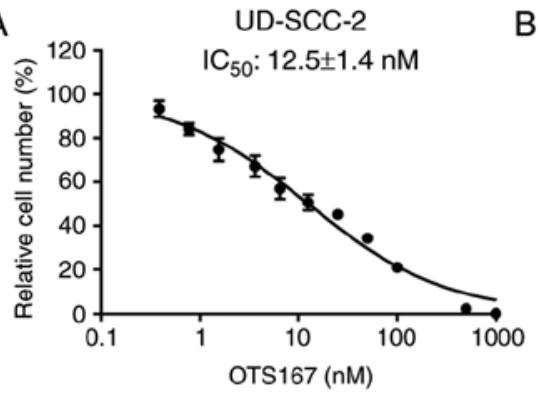

D

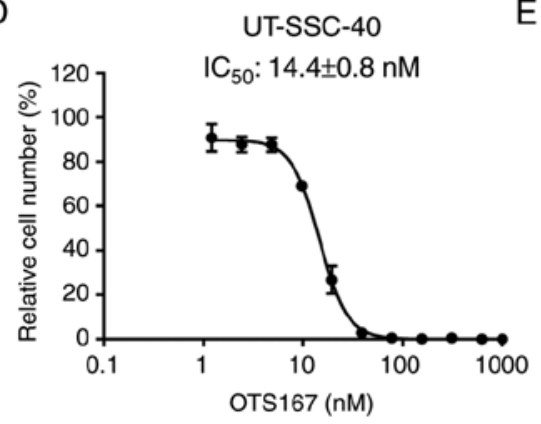

B

E
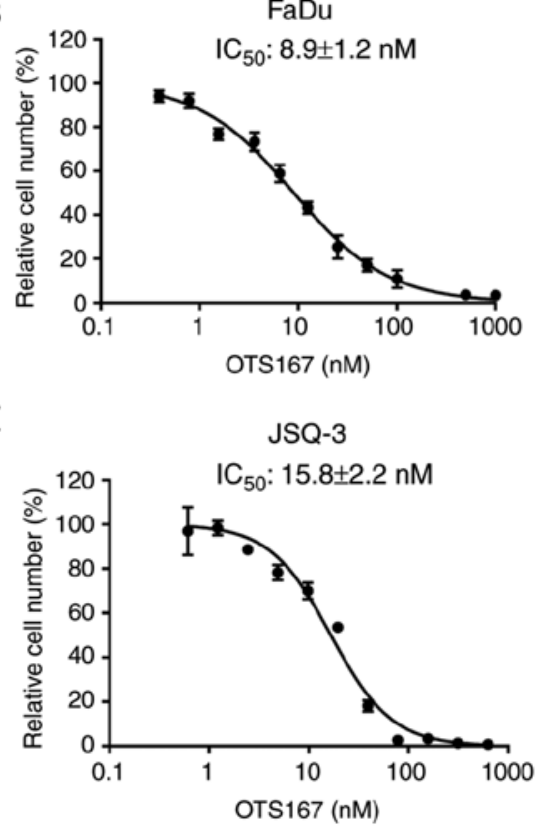

$\mathrm{HN}-5$

G

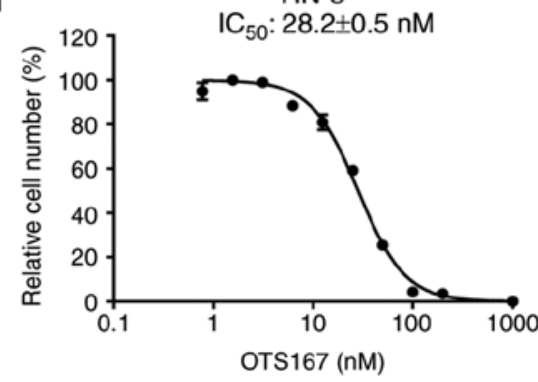

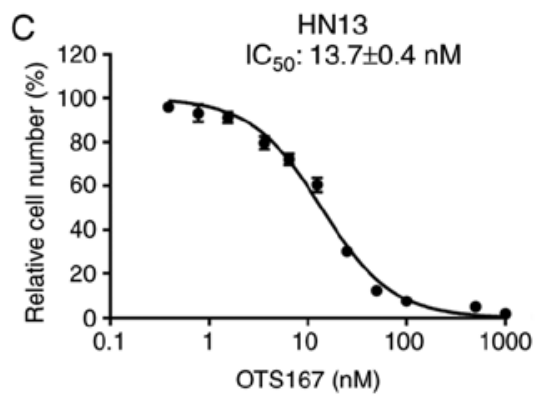

F

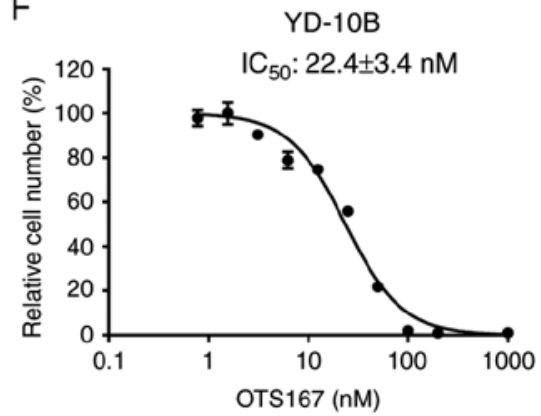

Figure 2. In vitro anti-proliferative effects of OTS167 in HNSCC cell lines. Each graph depicts growth inhibition curves of OTS167 for HNSCC cancer cell lines, (A) UD-SCC-2, (B) FaDu, (C) HN13, (D) UT-SCC-40, (E) JSQ-3, (F) YD-10B and (G) HN-5. HNSCC, head and neck squamous cell carcinoma; IC 50 half-maximum inhibitory concentration.

which exhibited relatively reduced $\mathrm{IC}_{50}$ values with a range of $8.9 \pm 1.2$ to $15.8 \pm 2.2 \mathrm{nM}$, compared with the two cell lines with reduced MELK expression, YD-10B and HN-5 cells, with $22.4 \pm 3.4$ and $28.2 \pm 0.5 \mathrm{nM}$, respectively (Fig. 2).

Knockdown with siRNA and OTS167 treatment could decrease SOX2 expression in HNSCC cells. Since SOX2 is considered as one of major stemness-associated genes $(10,11)$, whether MELK expression would affect the SOX2 expression levels in HNSCC cells was examined. Knockdown of MELK with siRNA decreased SOX2 expression in HN13 and UT-SCC-40 cells. At $48 \mathrm{~h}$ after siMELK transfection, the SOX2 expression level was significantly decreased, to $9.6 \pm 2.4 \%$, compared with HN13 cells transfected with control siRNA $(\mathrm{P}<0.001)$. However, UT-SCC-40 cells, which had reduced MELK expression, exhibited significant SOX2 downregulation (decreased to $43.4 \pm 4.8 \%$; $\mathrm{P}<0.001$ ), compared with the cells treated with control siRNA (Fig. 3A). These results indicated that SOX2 expression may be regulated by multiple factors, including MELK, which may be a major regulator of SOX2 expression in HN13 cells.

Additionally, HNSCC cells were treated with OTS167 at different concentrations (equivalent to $1 \mathrm{X}, 2 \mathrm{X}, 4 \mathrm{X}$ and $8 \mathrm{X} \mathrm{IC} \mathrm{C}_{50}$ concentrations) and it was identified that $\mathrm{SOX} 2$ expression was significantly downregulated in an OTS167 dose-dependent manner in the cell lines treated with OTS167 $(\mathrm{P}<0.0001$, compared with the cells treated with DMSO), as depicted in Fig. 3B. Due to MELK being a kinase and serving a critical role through the phosphorylation pathway (3-5), whether MELK could directly phosphorylate SOX2 was examined with an in vitro kinase assay using recombinant proteins.

In the in vitro kinase assay, without co-incubation with MELK recombinant protein, Histone $\mathrm{H} 3$ alone also exhibited a slight phosphorylation signal (Fig. 4; lane 3), which may be due to the contamination of unknown protein kinase(s) during the purification of recombinant Histone $\mathrm{H} 3$ protein. However, co-incubation of Histone $\mathrm{H} 3$ protein with MELK exhibited a strong phosphorylation signal (Fig. 4; lanes 6), indicating that the MELK recombinant protein could effectively phosphorylate the Histone $\mathrm{H} 3$ protein, which confirmed the efficiency of the in vitro kinase assay strategy. Furthermore, co-incubation of SOX2 with MELK demonstrated no phosphorylation signal (Fig. 4; lane 5), implying that SOX2 may not be a direct substrate of MELK. Due to SOX2 gene expression being suppressed at a similar level in two cell lines, OTS167 is considered to decrease SOX2 expression in UT-SCC-40 cells by another mechanism, such as an off-target effect (34). 
A
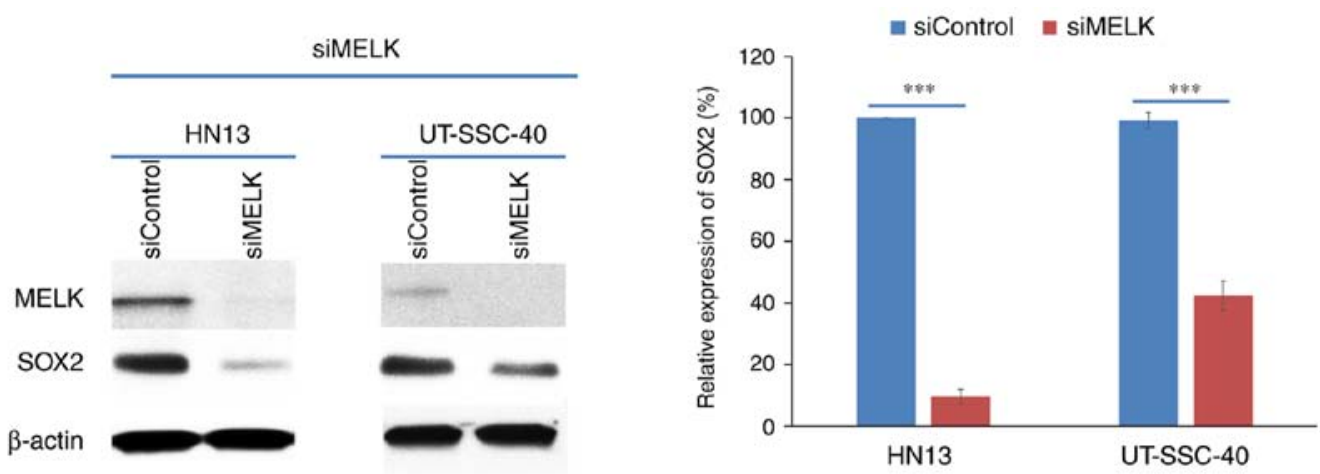

B

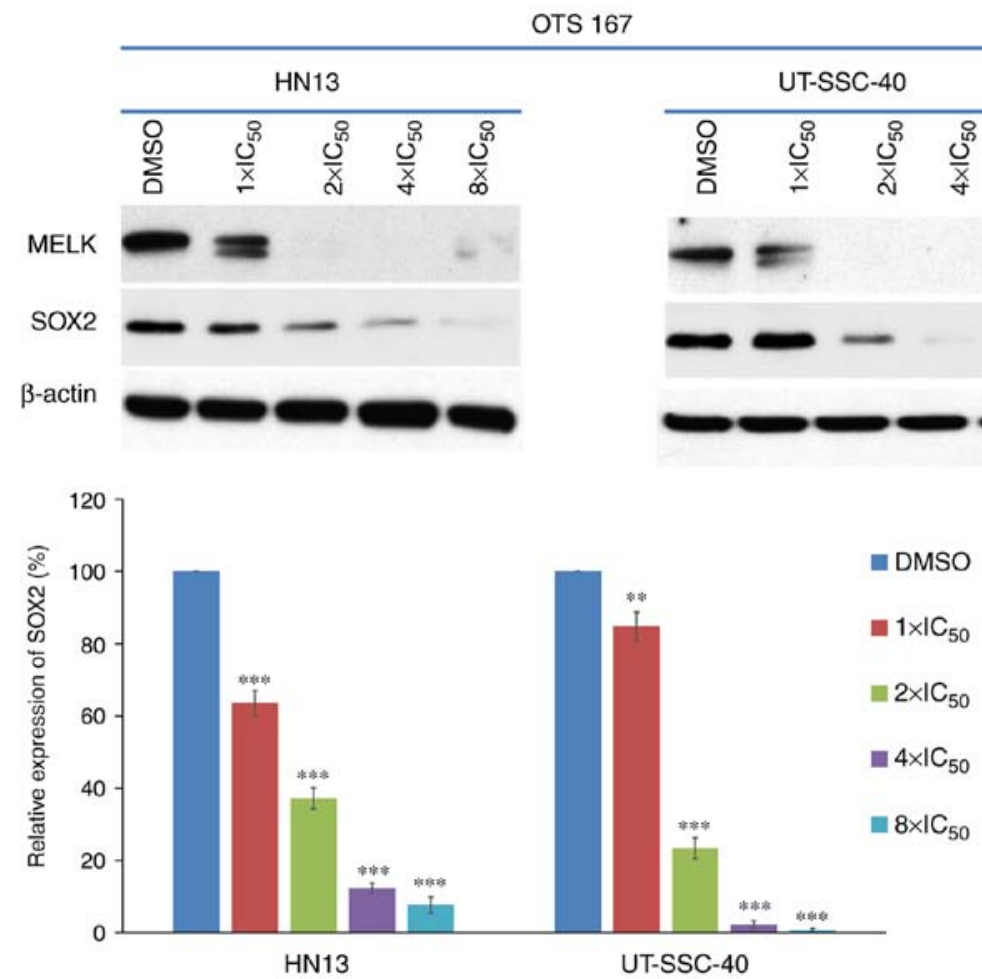

Figure 3. MELK inhibition affected SOX2 expression in HNSCC cell lines. (A) MELK knockdown by siRNA decreased SOX2 expression levels in HN13 and UT-SCC-40 cells. ${ }^{* * *} \mathrm{P}<0.001$, compared with the corresponding value of the siControl group. (B) MELK inhibitor OTS167 decreased SOX2 expression levels in a dose-dependent manner in HN13 and UT-SCC-40 cells. ${ }^{* * * *} \mathrm{P}<0.0001$, compared with the corresponding value of the DMSO-treated group. siRNA, small interfering RNA; MELK, maternal embryonic leucine zipper kinase; SOX2, SRY-box 2; DMSO, dimethyl sulfoxide; IC $_{50}$ half-maximum inhibitory concentration.

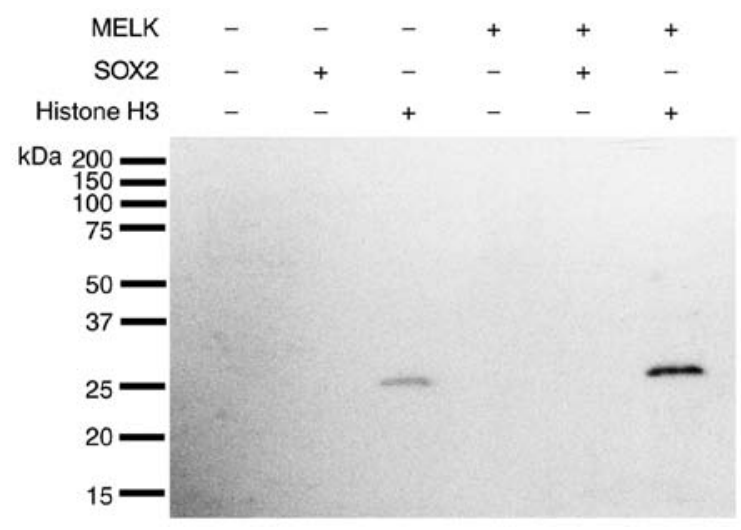

Figure 4. In vitro kinase assay of recombinant SOX2 with MELK recombinant protein. Recombinant Histone $\mathrm{H} 3$ protein was used as a positive control. Lane 1, no recombinant protein. Lane 2, only SOX2 protein. Lane 3, only Histone H3 protein. Lane 4, only MELK. Lane 5, co-incubation of SOX2 with MELK. Lane 6, co-incubation of Histone H3 protein with MELK. MELK, maternal embryonic leucine zipper kinase; SOX2, SRY-box 2.
Knockdown of MELK decreases a series of TFS of SOX2. To investigate a mechanism for SOX2 downregulation by MELK inhibition, possible TFs of SOX2 were examined. SOX2 expression is reported to be exquisitely controlled in mammals and it may be regulated by a large number of TFs, including serine racemase 1 (SRR1) and SRR2 and forkhead box $\mathrm{O} 1$ (35). Therefore, a promoter-binding TF profiling assay was performed to screen TFs that could bind to the promoter region of SOX2 in HN13 cells. In the assay, the synthesized DNA corresponding to a SOX2 promoter region was mixed with a set of 96 biotin-labelled oligonucleotides corresponding to 96 TFs along with nuclear extract of cells. When the SOX2 promoter DNA competes with the biotin-labelled oligonucleotide for the binding to TFs in the nuclear extract, it causes no or reduced detection of luminescent signals when the TF plate hybridized with eluted probes. The decreasing fold of luminescent signals with or without the SOX2 promoter DNA fragment was calculated 
A

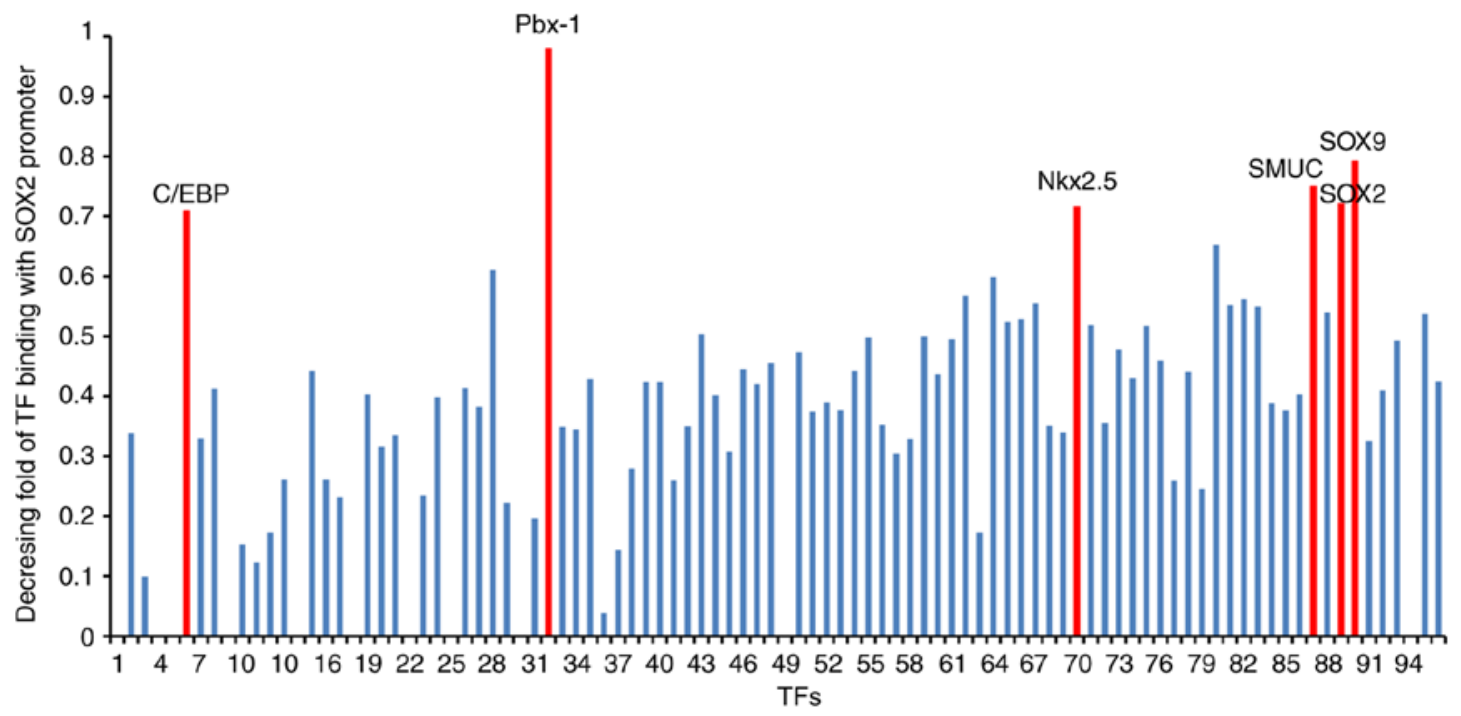

B

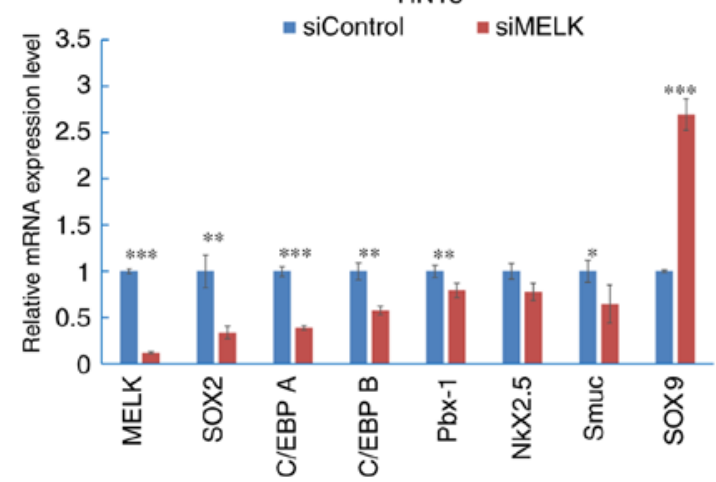

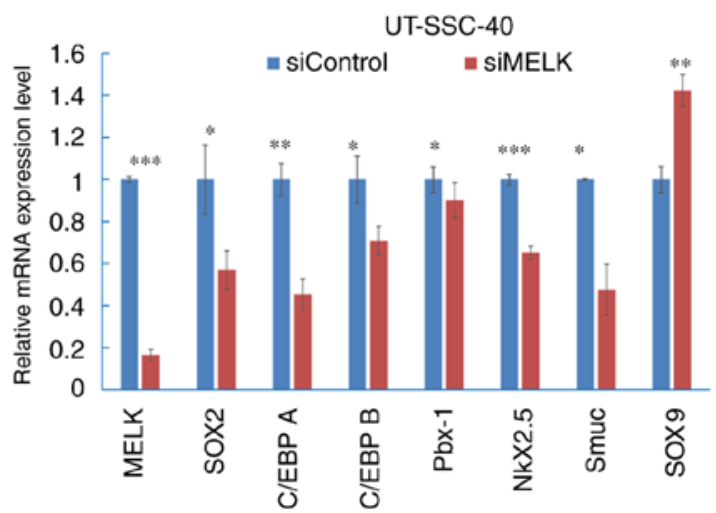

Figure 5. SOX2 TFs analysis. (A) Screening of SOX2 TFs. Red bars represent TFs that could bind to a SOX2 promoter region sequence. (B) Reverse transcription-quantitative polymerase chain reaction results of SOX2 TF candidates following MELK inhibition. ${ }^{*} \mathrm{P}<0.05,{ }^{* * *} \mathrm{P}<0.01,{ }^{* * * *} \mathrm{P}<0.001$, compared with the corresponding value of the siControl group. siRNA, small interfering RNA; MELK, maternal embryonic leucine zipper kinase; TF, transcription factor; SOX2, SRY-box 2; C/EBP, CCAAT enhancer binding protein; SMUC, snail family transcription repressor 3.

and TFs with a greater decreasing fold $(>0.7)$ were selected. CCAAT enhancer binding protein (C/EBP), Pbx1, Nkx2.5, snail family transcription repressor 3 (SMUC), SOX2 and SOX9 were considered to bind strongly to the SOX2 promoter region in the cancer cells examined (Fig. 5A). RT-qPCR was then performed to quantify the expression levels of TF candidates in MELK-knockdown cells and the control cells, and it was determined that following knockdown of MELK in HN13 cells, SOX2 was downregulated to $0.34 \pm 0.06(\mathrm{P}<0.01)$, compared with the siControl group $(1 \pm 0.17)$; C/EBP A was downregulated to $0.39 \pm 0.02(\mathrm{P}<0.001)$, compared with the siControl group $(1 \pm 0.05)$; C/EBP B was downregulated to $0.58 \pm 0.04(\mathrm{P}<0.01)$, compared with the siControl

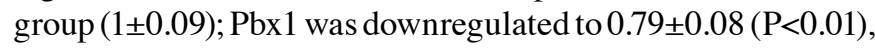
compared with the siControl group $(1 \pm 0.06)$; $\mathrm{Nkx} 2.5$ was downregulated to $0.78 \pm 0.09$ ( $\mathrm{P}>0.05)$, compared with the siControl group $(1 \pm 0.08)$; and SMUC were downregulated to $0.65 \pm 0.02(\mathrm{P}<0.05)$, compared with the siControl group (1 \pm 0.12$)$. In UT-SCC-40 cells, SOX2 was downregulated to $0.57 \pm 0.09(\mathrm{P}<0.05)$, compared with the siControl group $(1 \pm 0.16) ; \mathrm{C} / \mathrm{EBP} \mathrm{A}$ was downregulated to $0.45 \pm 0.07(\mathrm{P}<0.01)$, compared with the siControl group $(1 \pm 0.07)$; $\mathrm{C} / \mathrm{EBP} \mathrm{B}$ was downregulated to $0.71 \pm 0.06(\mathrm{P}<0.05)$, compared

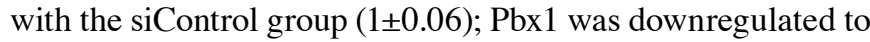
$0.90 \pm 0.08(\mathrm{P}<0.05)$, compared with the siControl group $(1 \pm 0.08)$; $\mathrm{Nkx} 2.5$ was downregulated to $0.65 \pm 0.03(\mathrm{P}<0.001)$, compared with the siControl group $(1 \pm 0.03)$; and SMUC were downregulated to $0.48 \pm 0.12(\mathrm{P}<0.05)$ compared with the siControl

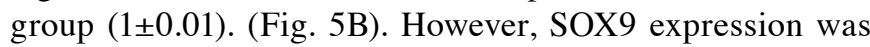
significantly increased in the cells, compared with the control cells $(\mathrm{P}<0.001$ in $\mathrm{HN13}, \mathrm{P}<0.01$ in UT-SCC40).

\section{Discussion}

It was previously reported that MELK could be a promising target for cancer therapy and developed its potent inhibitor, OTS167, which could effectively suppress MELK activity and inhibit the phosphorylation of various MELK substrates, including B-cell lymphoma 2 like 14, cell division cycle 25B, mitogen-activated protein kinase kinase kinase 5 and zinc finger protein $622(4,5)$. It has to date been identified that OTS167 exhibited significant suppressive effects on various cancer cell lines, including breast cancer, kidney cancer, small cell lung cancer (SCLC) and multiple myeloma (4,5,29,31-33). Additionally, it was demonstrated that OTS167 could target CSCs and suppress the mammosphere formation of cancer 
stem-like cells $(4,5)$. In a number of SCLC cell lines, it was determined that OTS167 treatment could induce cytokinetic defects with intercellular bridges and the formation of neuronal protrusions accompanied with an increase of a neuronal differentiation markerx (cycle of differentiation 56), indicating that this compound may induce differentiation of cancer stem-like cells to neuron-like cells (33).

In the present study, it was demonstrated that HNSCC cells with increased MELK expression had an improved ability to produce large spheres under the serum-free conditions. It was then revealed that MELK inhibition by siRNA and OTS167 could significantly downregulate SOX2 expression in HNSCC cells and it was determined that the expression levels of a set of SOX2 transcriptional factors were decreased following MELK inhibition. This indicated that OTS167 may target CSCs through the downregulation of SOX2. Notably, it was previously reported in a breast cancer MDA-MB-231 cell line, which has undifferentiated, cancer stem-like characteristics, that snail family transcriptional repressor 2 (Slug), a CSC marker, was determined to be reduced with OTS167 treatment in a dose-dependent manner (5). Slug expression increased SOX2 and Nanog expression and promoted the progression of hepatocellular cancer. Knockdown of Slug with siRNA notably reduced SOX2 and Nanog expression and resulted in inhibition of hepatocellular carcinoma cell migration in vitro (36). These data indicated that there may be an association between MELK, Slug and SOX2.

However, the present study has a number of limitations, which should be addressed in the future. First of all, although it was demonstrated that MELK inhibition affected the expression levels of transcriptional factors for SOX2, the detailed mechanism was not clarified. It would be beneficial to examine the TF profiles with a chromatin immunoprecipitation assay to further define the SOX2 TFs in these cell lines. The commercial phosphorylated (p)-SOX2 or p-histone generally only targets a number of specific sites of the proteins. However, in the present study, there was no information available regarding the sites of SOX2 that could be phosphorylated by MELK. Phosphorylation frequently occurs at Ser/Thr or Tyr sites; therefore, the universal antibody (anti-phospho-Ser/Thr/Tyr antibody) was used to include as many phosphorylation sites as possible. Furthermore, additional analyses regarding how MELK inhibition would affect the stemness, migration and invasion of these cancer cells should also be performed to obtain comprehensive information for the clinical application of OTS167.

Although further investigation is necessary, the present data demonstrates that MELK inhibition could target cancer stemness through the suppression of SOX2 expression. It was reported that partial suppression of SOX2 expression reduced the cell viability, sphere formation, clonal growth as well as tumorigenicity in multiple cancer types, including glioblastoma, small-cell lung cancer and numerous forms of squamous cell carcinoma (37-39). Considering the ability to target CSCs as well as cancer cells, the MELK inhibitor may be a promising drug in clinical applications for HNSCC.

\section{Acknowledgements}

The authors would like to thank Dr Tanguy Y. Seiwert (Department of Medicine, University of Chicago, Chicago,
IL, USA), who provided the HNSCC cell lines (UD-SCC-2, UT-SCC-40, HN-SCC-151, FaDu, JSQ-3, HN-5, HN-6 and HN13) for this research.

\section{Funding}

This study was supported by a research grant from OncoTherapy Science Inc. and Science and Technology Planning Project of Guangdong Province (grant no. 2016A020215207).

\section{Availability of data and materials}

The datasets used and/or analyzed during the present study are available from the corresponding author on reasonable request.

\section{Authors' contributions}

YN designed and supervised the project and edited the manuscript. LR performed the majority of the experiments and wrote the manuscript. BD assisted in the experiments and data analysis. VS set up and assisted with the sphere formation experiment and the culture of cancer cell lines. JHP directed and supervised the techniques, designed the project and was involved in the data analysis. All authors read and approved the manuscript and agree to be accountable for all aspects of the research in ensuring that the accuracy or integrity of any part of the work are appropriately investigated and resolved.

\section{Ethics approval and consent to participate}

Not applicable.

\section{Patient consent for publication}

Not applicable.

\section{Competing interests}

YN is a stock holder and a scientific advisor of OncoTherapy Science, Inc. JP is a scientific advisor of OncoTherapy Science, Inc. Oncotherapy Science, Inc., also provided financial support and certain of the reagents for this study. No potential conflicts of interest were disclosed by the other authors.

\section{References}

1. Global Burden of Disease Cancer Collaboration, Fitzmaurice C, Allen C, Barber RM, Barregard L, Bhutta ZA, Brenner H, Dicker DJ, Chimed-Orchir O, Dandona R, et al: Global, regional, and national cancer incidence, mortality, years of life lost, years lived with disability, and disability-adjusted life-years for 32 cancer groups, 1990 to 2015: A systematic analysis for the global burden of disease study. JAMA Oncol 3: 524-548, 2017.

2. Chaturvedi AK, Anderson WF, Lortet-Tieulent J, Curado MP, Ferlay J, Franceschi S, Rosenberg PS, Bray F and Gillison ML: Worldwide trends in incidence rates for oral cavity and oropharyngeal cancers. J Clin Oncol 31: 4550-4559, 2013.

3. Ganguly R, Hong CS, Smith LG, Kornblum HI and Nakano I: Maternal embryonic leucine zipper kinase: Key kinase for stem cell phenotype in glioma and other cancers. Mol Cancer Ther 13: 1393-1398, 2014.

4. Chung S, Suzuki H, Miyamoto T, Takamatsu N, Tatsuguchi A, Ueda K, Kijima K, Nakamura Y and Matsuo Y: Development of an orally-administrative MELK-targeting inhibitor that suppresses the growth of various types of human cancer. Oncotarget 3: 1629-1640, 2012. 
5. Chung S and Nakamura Y: MELK inhibitor, novel molecular targeted therapeutics for human cancer stem cells. Cell Cycle 12: $1655-1656,2013$

6. ClinicalTrials.gov: Phase 1 study of OTS167 in patients with solid tumors. https://clinicaltrials.gov/ct2/show/NCT01910545. Accessed July 29, 2013.

7. ClinicalTrials.gov: Pharmacological study of intravenous OTS167 in patients with refractory or relapsed acute myeloid leukemia acute lymphoblastic leukemia, advanced myelodysplastic syndromes, advanced myeloproliferative neoplastic disorders, or advanced chronic myelogenous leukemia. https://clinicaltrials. gov/ct2/show/NCT02795520. Accessed June 10, 2016.

8. ClinicalTrials.gov: Safety study of MELK inhibitor to treat patients with advanced breast cancer and triple negative breast cancer. https://clinicaltrials.gov/ct2/show/NCT02926690. Accessed October 6, 2016.

9. Prince ME, Sivanandan R, Kaczorowski A, Wolf GT, Kaplan MJ, Dalerba P, Weissman IL, Clarke MF and Ailles LE: Identification of a subpopulation of cells with cancer stem cell properties in head and neck squamous cell carcinoma. Proc Natl Acad Sci USA 104: 973-978, 2007.

10. Weina K and Utikal J: SOX2 and cancer: Current research and its implications in the clinic. Clin Transl Med 3: 19, 2014.

11. Herreros-Villanueva M,Zhang JS, Koenig A, AbelEV, Smyrk TC, Bamlet WR, de Narvajas AA, Gomez TS, Simeone DM, Bujanda L and Billadeau DD: SOX2 promotes dedifferentiation and imparts stem cell-like features to pancreatic cancer cells. Oncogenesis 2: e61, 2013.

12. Bareiss PM, Paczulla A, Wang H, Schairer R, Wiehr S, Kohlhofer U, Rothfuss OC, Fischer A, Perner S, Staebler A, et al: SOX2 expression associates with stem cell state in human ovarian carcinoma. Cancer Res 73: 5544-5555, 2013.

13. Prior HM and Walter MA: SOX genes: Architects of development. Mol Med 2: 405-412, 1996.

14. Stevanovic M, Zuffardi O, Collignon J, Lovell-Badge R and Goodfellow P: The cDNA sequence and chromosomal location of the human SOX2 gene. Mamm Genome 5: 640-642, 1994

15. Cai YR, Zhang HQ, Qu Y, Mu J, Zhao D, Zhou LJ, Yan H, Ye JW and Liu Y: Expression of MET and SOX2 genes in non-small cell lung carcinoma with EGFR mutation. Oncol Rep 26: 877-885, 2011

16. Gen Y, Yasui K, Nishikawa T and Yoshikawa T: SOX2 promotes tumor growth of esophageal squamous cell carcinoma through the AKT/mammalian target of rapamycin complex 1 signaling pathway. Cancer Sci 104: 810-816, 2013.

17. Yang Z, Pan X, Gao A and Zhu W: Expression of Sox 2 in cervical squamous cell carcinoma. J BUON 19: 203-206, 2014

18. Sun C, Sun L, Li Y, Kang X, Zhang S and Liu Y: Sox2 expression predicts poor survival of hepatocellular carcinoma patients and it promotes liver cancer cell invasion by activating slug. Med Oncol 30: 503, 2013

19. Lengerke C, Fehm T, Kurth R, Neubauer H, Scheble V, Müller F, Schneider F, Petersen K, Wallwiener D, Kanz L, et al: Expression of the embryonic stem cell marker SOX2 in early-stage breast carcinoma. BMC Cancer 11: 42, 2011

20. Lee SH, Oh SY, Do SI, Lee HJ, Kang HJ, Rho YS, Bae WJ and Lim YC: SOX2 regulates self-renewal and tumorigenicity of stem-like cells of head and neck squamous cell carcinoma. Br J Cancer 111: 2122-2130, 2014.

21. Luo W, Li S, Peng B, Ye Y, Deng X and Yao K: Embryonic stem cells markers SOX2, OCT4 and Nanog expression and their correlations with epithelial-mesenchymal transition in nasopharyngeal carcinoma. PLoS One 8: e56324, 2013.

22. Ge N, Lin HX, Xiao XS, Guo L, Xu HM, Wang X, Jin T, Cai XY, Liang Y, Hu WH, et al: Prognostic significance of Oct4 and Sox2 expression in hypopharyngeal squamous cell carcinoma. J Transl Med 8: 94,2010

23. Wang X, Liang Y, Chen Q, Xu HM, Ge N, Luo RZ, Shao JY, He Z, Zeng YX, Kang T, et al: Prognostic significance of SOX2 expression in nasopharyngeal carcinoma. Cancer Invest 30: $79-85,2012$.
24. Du L, Yang Y, Xiao X, Wang C, Zhang X, Wang L, Zhang X, Li W, Zheng G, Wang S, et al: Sox2 nuclear expression is closely associated with poor prognosis in patients with histologically node-negative oral tongue squamous cell carcinoma. Oral Oncol 47: 709-713, 2011.

25. Dai W, Tan X, Sun C and Zhou Q: High expression of SOX2 is associated with poor prognosis in patients with salivary gland adenoid cystic carcinoma. Int J Mol Sci 15: 8393-8406, 2014.

26. Tang XB, Shen XH, Li L, Zhang YF and Chen GQ: SOX2 overexpression correlates with poor prognosis in laryngeal squamous cell carcinoma. Auris Nasus Larynx 40: 481-486, 2013.

27. Gonzalez-MarquezR,Llorente JL,RodrigoJP,Garcia-PedreroJM, Alvarez-Marcos C, Suarez C and Hermsen MA: SOX2 expression in hypopharyngeal, laryngeal, and sinonasal squamous cell carcinoma. Hum Pathol 45: 851-857, 2014.

28. Dong Z, Liu G, Huang B, Sun J and Wu D: Prognostic significance of SOX2 in head and neck cancer: A meta-analysis. Int J Clin Exp Med 7: 5010-5020, 2014.

29. Chung S, Kijima K, Kudo A, Fujisawa Y, Harada Y, Taira A, Takamatsu N, Miyamoto T, Matsuo Y and Nakamura Y: Preclinical evaluation of biomarkers associated with antitumor activity of MELK inhibitor. Oncotarget 7: 18171-18182, 2016.

30. Livak KJ and Schmittgen TD: Analysis of relative gene expression data using real-time quantitative PCR and the $2^{-\Delta \Delta C \mathrm{~T}}$ method. Methods 25: 402-408, 2001.

31. Inoue H, Kato T, Olugbile S, Tamura K, Chung S, Miyamoto T, Matsuo Y, Salgia R, Nakamura Y and Park JH: Effective growth-suppressive activity of maternal embryonic leucinezipper kinase (MELK) inhibitor against small cell lung cancer. Oncotarget 7: 13621-13633, 2016.

32. Kato T, Inoue H, Imoto S, Tamada Y, Miyamoto T, Matsuo Y, Nakamura Y and Park JH: Oncogenic roles of TOPK and MELK, and effective growth suppression by small molecular inhibitors in kidney cancer cells. Oncotarget 7: 17652-17664, 2016.

33. Stefka AT, Park JH, Matsuo Y, Chung S, Nakamura Y, Jakubowiak AJ and Rosebeck S: Anti-myeloma activity of MELK inhibitor OTS167: Effects on drug-resistant myeloma cells and putative myeloma stem cell replenishment of malignant plasma cells. Blood Cancer J 6: e460, 2016.

34. Lin A, Giuliano CJ, Sayles NM and Sheltzer JM: CRISPR/Cas9 mutagenesis invalidates a putative cancer dependency targeted in on-going clinical trials. Elife 6: e24179, 2017.

35. Wuebben EL and Rizzino A: The dark side of SOX2: Cancer - A comprehensive overview. Oncotarget 8: 44917-44943, 2017.

36. Zhao X, Sun B, Sun D, Liu T, Che N, Gu Q, Dong X, Li R, Liu Y and Li J: Slug promotes hepatocellular cancer cell progression by increasing sox2 and nanog expression. Oncol Rep 33: 149-156, 2015.

37. Jia X, Li X, Xu Y, Zhang S, Mou W, Liu Y, Liu Y, Lv D, Liu CH, Tan X, et al: SOX2 promotes tumorigenesis and increases the anti-apoptotic property of human prostate cancer cell. J Mol Cell Biol 3: 230-238, 2011.

38. Tian Y, Jia X, Wang S, Li Y, Zhao P, Cai D, Zhou Z, Wang J, Luo $\mathrm{Y}$ and Dong M: SOX2 oncogenes amplified and operate to activate AKT signaling in gastric cancer and predict immunotherapy responsiveness. J Cancer Res Clin Oncol 140: 1117-1124, 2014.

39. Sanada Y, Yoshida K, Ohara M, Oeda M, Konishi K and Tsutani Y: Histopathologic evaluation of stepwise progression of pancreatic carcinoma with immunohistochemical analysis of gastric epithelial transcription factor $S$ : Comparison of expression patterns between invasive components and cancerous or nonneoplastic intraductal components. Pancreas 32: 164-170, 2006. 